Gynäkologe 2016 • 49:437-441

DOI 10.1007/s00129-016-3883-4

Online publiziert: 17. Mai 2016

(c) The Author(s) 2016. This article is available

at SpringerLink with Open Access

\section{Redaktion}

A. Schröer, Berlin

CrossMark

\section{Maximilian Schmid}

Abteilung für Geburtshilfe und feto-maternale Medizin, Universitätsklinik für Frauenheilkunde, Medizinische Universität Wien, Allgemeines Krankenhaus der Stadt Wien, Wien, Österreich

\title{
Nichtinvasive pränatale Tests in der Zukunft
}

\section{Ausweitung der Indikationen, Evolution der Untersuchungsplattform und Regionalisierung}

\section{Nichtinvasive Pränataltests - ein neues Screeningparadigma}

Die vollständige Sequenzierung des menschlichen Genoms im Jahr 2001 und der enorme technische Fortschritt haben $\mathrm{zu}$ einer Revolution in der genetischen Diagnostik geführt. Nichtinvasive Pränataltests (NIPT), genauer auch cfDNA (zellfreie DNA)-Tests genannt, sind seit 2011 klinisch verfügbar und haben die pränatale Diagnostik entscheidend verändert. Sie ermöglichen eine höchst zuverlässige Beurteilung des Risikos für häufige Chromosomenstörungen beim Feten. Die Tests beruhen auf der seit längerem bekannten Tatsache, dass im mütterlichen Blut genetisches Material (cfDNA) sowohl der Mutter als auch des Feten vorhanden ist. Dieses wird mittels hochentwickelter Methoden zur DNA-Analyse (z. B. Next Generation Sequencing, NGS; Microarray-Analyse) untersucht. Über Messung der Konzentration und der Verteilung der cfDNA wird eine Einschätzung abgeleitet, ob das ungeborene Kind von einer Chromosomenstörung betroffen sein könnte oder nicht.

CfDNA-Tests haben eine hervorragende Sensitivität und Spezifität beim Screening für Trisomie 21 (Down-Syndrom) und hier eine höhere Aussagekraft als das herkömmliche Ersttrimesterscreening (ETS; Combined-Test). Klinisch steht dabei besonders die Möglichkeit der Reduktion unnötiger invasiver
Eingriffe nach falsch-auffälligem ETS im Vordergrund. Dies beruht auf der Tatsache, dass der Combined-Test mit $5 \%$ eine deutlich höhere Falsch-positiv-Rate hat als cfDNA-Tests mit $<0,1 \%$ [1]. Mittlerweile konnte auch belegt werden, dass der Einsatz von cfDNA-Tests als primäre Screeningmethode klinisch sinnvoll ist. Auf diese Weise können theoretisch Erkennungsraten von $>99 \%$ für Trisomie 21 bei einer Falsch-positiv-Rate von $<0,1 \%$ erreicht werden [2]. Es ist also zu erwarten, dass die Bestimmung der cfDNA aus dem mütterlichen Blut den Combined-Test beim Screening auf Trisomie 21 in naher Zukunft ablöst.

\section{》) CfDNA-Tests helfen bei der Reduktion unnötiger invasiver Diagnostik}

Es ist auch bereits gelungen, das gesamte fetale Genom mittels cfDNA-Analyse aus dem mütterlichen Blut $\mathrm{zu}$ sequenzieren $[3,4]$, und kommerzielle Anbieter bieten bereits ein genomweites Screening auf Chromosomenaberration $>7$ Megabasen $(\mathrm{Mb})$ an. Darüber hinaus gibt es in der Labortechnik deutliche Fortschritte. War die Durchführung von cfDNA-Tests primär auf einige NGS-Labors in den USA und China beschränkt, wird NIPT demnächst in den meisten industrialisierten Ländern regional durchgeführt werden. Neue kostengünstigere und einfachere Plattformen zur cfDNA-Analyse werden nicht nur die Regionalisierung weiter fördern, sondern auch die Verbreitung von NIPT bei schwangeren Frauen deutlich steigern. Im Folgenden werden diese Entwicklungen näher beschrieben und kritisch hinterfragt.

\section{Ausweitung von Indikationen für NIPT}

Zahlreiche Studien belegen, dass cfDNATests eine in dieser Form bis dato nicht erreichbare, zuverlässige Beurteilung des Risikos für die „klassischen“ Trisomien (i. e. Trisomie 21, 18 und 13) beim Feten ermöglichen [1]. Der Wunsch vieler schwangerer Frauen nach einem ,gesunden Kind“, in Kombination mit der theoretischen Möglichkeit der Sequenzierung des gesamten fetalen Genoms aus dem mütterlichen Blut spornt kommerzielle Anbieter dazu an, NIPT auf möglichst viele genetische Krankheitsbilder auszudehnen. Diese Entwicklung ist jedoch kritisch zu hinterfragen, da diese Ausweitung meist ohne ausreichende wissenschaftliche Evidenz geschieht, sie zu einer deutlichen Zunahme der (kumulativen) Falsch-positiv-Rate (FPR) von NIPT führt und da oft vergessen wird, dass kein genetisches Testverfahren eine Garantie auf ein gesundes Kind geben kann.

Fast alle kommerziellen Anbieter von cfDNA-Tests haben begonnen, die Indikationen für NIPT auf bisher unter Gynäkologen wenig bekannte geneti- 


\begin{tabular}{|lllll}
\hline Erkrankung & Chromosom & Prävalenz & Größe & \% mit Deletion $\geq 3 \mathbf{~ M b ~ ( i n ~ \% ) ~}$ \\
\hline DiGeorge-Syndrom & $22 q 11.2$ & $\begin{array}{l}1: 2000[17] \\
1: 4000[18]\end{array}$ & $3 \mathrm{Mb}$ (häufigste Deletion) [19] & 85 \\
& & $\begin{array}{l}1: 5000 \\
1: 10.000[20]\end{array}$ & $1,5-10,5 \mathrm{Mb}[21,22]$ & 85 \\
\hline Monosomie 1p36 & $1 \mathrm{p} 36$ & $1: 20.000$ & $\begin{array}{l}5 \mathrm{Mb} \text { und 6 Mb (häufige Deletionen) } \\
{[23,24]}\end{array}$ & $70[23,24]$ \\
\hline $\begin{array}{llll}\text { Angelman-Syndrom oder Prader-Willi } \\
\text { Syndrome }\end{array}$ & $15 q 11.2-q 13$ & $1: 50.000[25]$ & $10-30 \mathrm{Mb}[26,27]$ & $99[26,27]$ \\
\hline Cri-du-Chat-Syndrom & $5 p 15$ & & & \\
\hline
\end{tabular}

sche Indikationen bzw. Krankheitsbilder auszudehnen. Primär wurde zusätzlich die Möglichkeit zum Screening auf Störungen der Geschlechtschromosomen (z. B. Turner-Syndrom, Klinefelter-Syndrom) angeboten. Diese Anwendung ist bis heute höchst umstritten und wird derzeit in Deutschland, Österreich und der Schweiz von renommierten Fachgesellschaften nicht uneingeschränkt empfohlen [5]. Die Literatur zeigt auch, dass die Performance bei dieser Indikation, unabhängig von Anbieter und Verfahren, deutlich unter jener für Trisomie 21, 18 und 13 liegt. Darüber hinaus gestaltet sich die Beratung schwierig, insbesondere bei Vorliegen eines auffälligen cfDNA-Tests, nicht zuletzt, weil der klinische Phänotyp bei Störungen der Geschlechtschromosomen sehr variabel ist. Viele Betroffene leiden, wenn überhaupt, nur unter leichten Störungen der physischen oder psychischen Entwicklung.

$\mathrm{Zu}$ beachten ist, dass das Hinzufügen neuer Indikationen bzw. Krankheitsbilder die kumulierte Falsch-positivRate von NIPT signifikant ansteigen lässt. Dadurch wird ein wesentlicher Vorteil gegenüber dem ETS zunichte gemacht. Dies gilt insbesondere für seltene Mikrodeletionssyndrome. Die meisten kommerziellen Anbieter von cfDNA-Tests bieten bereits „Panels“ für immer mehr Mikrodeletionssyndrome an. Darin enthalten ist zumeist eine cfDNA-Analyse auf Vorliegen einer Deletion 22q11.2 (DiGeorge-Syndrom), einer Deletion 1p36 und 15q11.2-q13 (assoziiert mit dem Prader-Willi und dem Angelmann-Syndrom), sowie einer terminalen Deletion auf Chromosom $5 p$ (Cri-du-Chat-Syndrom) und Chromosom 4p (Wolf-Hirsch-Syndrom). Die Prävalenzen dieser Erkrankungen sind in • Tab. 1 angeführt. Der Autor sieht die Anwendung cfDNA-Tests zum Screening auf Mikrodeletionssyndrome vor allem kritisch, weil die geringe Prävalenz der meisten dieser Syndrome dazu führt, dass selbst bei hervorragender Spezifität vor allem falsch-positive cfDNATestergebnisse generiert werden [6].

Eine Ausnahme könnte hier das Mikrodeletionssyndrom 22q11.2 (DiGeorge-Syndrom) darstellen. Rezente Daten sprechen dafür, dass diese Erkrankung mit einer Prävalenz von 1:1000 bei Feten wesentlich häufiger ist als ursprünglich angenommen [7]. Somit könnte diese Chromosomenstörung bei Feten junger schwangerer Frauen wesentlich häufiger sein als die Trisomie 21. Dennoch fehlt derzeit eine solide klinische Evidenz zum Einsatz von NIPT für diese Indikation. Leistungsdaten wurden bis jetzt aus einer extrem kleinen Anzahl von Proben abgeleitet, die vornehmlich in vitro erzeugt wurden, bzw. beschränken sich auf retrospektive Analysen kommerzieller Proben ohne adäquates Follow-up [8, 9]. Die Performance als Screeningverfahren in einer allgemeinen Schwangerenpopulation ist daher derzeit unbekannt und der klinische Nutzen umstritten.

\section{》) Chromosomenstörungen $<3 \mathrm{Mb}$ werden überwiegend nicht erkannt}

Auch ein sicherer Ausschluss von Mikrodeletionssyndromen bei Patienten mit hohem Risiko (z. B. Feten mit Fehlbildungen) mittels cfDNA-Analyse ist nicht möglich. Es handelt sich bei NIPT generell ja um Screening und nicht um ein diagnostisches Verfahren (falsch-negative Befunde sind möglich). Darüber hinaus beschränken sich cfDNA-Tests generell auf einige wenige Mikrodeletionssyndrome. Somit bleibt selbst bei unauffälligem cfDNA-Test das Hintergrundrisiko für die Gesamtheit dieser Erkrankungen im Wesentlichen unverändert. In diesem Zusammenhang ist es wichtig zu erwähnen, dass die Performance äußerst abhängig von der Größe der Deletion ist und Chromosomenstörungen $<3 \mathrm{Mb}$ überwiegend nicht erkannt werden (z. B. ca. $15 \%$ der Fälle von Mikrodeletionssyndrom 22q11.2).

\section{》) Die Analyse des gesamten fetalen Genoms wirft ethische Fragen auf}

Es gibt auch bereits erfolgreiche Ansätze, monogenetische Erkrankungen, z. B. die zystische Fibrose, beim Feten mittels cfDNA-Tests zu erkennen. Diese Anwendung von NIPT ist aber derzeit als rein experimentell zu betrachten und nur im Rahmen klinischer Studien zu befürworten [10]. Eine Analyse des gesamten fetalen Genoms aus mütterlichem Blut ist nicht nur technisch äußerst komplex, extrem teuer und bis jetzt nur in Einzelfällen gelungen, sondern wirft vor allem viele ethische Fragen auf, die derzeit wohl kaum zu beantworten sind. Eine genaue Diskussion dazu, wie z. B. von Skirton et al. [11] dargelegt, liegt jenseits der Möglichkeiten dieses Übersichtsartikels.

\section{Evolution der cfDNA- Untersuchungsplattform}

In den letzten Jahren wurden innovative Verfahren der DNA-Sequenzierung entwickelt, die unter dem Begriff NGS zusammengefasst werden. Diese Technolo- 
gie beruht auf dem Prinzip der massiven parallelen Sequenzierung von Millionen DNA-Fragmenten in einem einzigen Sequenzierungslauf. Im Vergleich zur konventionellen DNA-Sequenzierung nach Sanger ist die Kapazität also um mehr als den Faktor 1000 höher (Hochdurchsatzsequenzierung). NGS ermöglicht so eine rasche Identifizierung von cfDNAFragmenten, die bisher aus Kapazitätsund Kostengründen nicht möglich war [12]. Erst durch NGS wurde NIPT in der uns bekannten Form überhaupt möglich.

Ende 2014 gelang es Wissenschaftlern erstmals, eine DNA-MicroarrayQuantifizierungsmethode für NIPT erfolgreich zu implementieren. Sie konnten mit diesem Verfahren signifikante Vorteile im Vergleich zum bisherigen Sequenzierungsansatz erzielen. Juneau et al. [13] konnten zeigen, dass die Microarray-Technologie unter Umständen eine genauere cfDNA-Analyse als die Hochdurchsatzsequenzierung erlaubt, schneller Ergebnisse liefert und außerdem kostengünstiger ist. Seitdem wird weiter fieberhaft an Alternativen zur immer noch teuren, technisch aufwendigen und meist auch zeitintensiven NGS-Technologie gesucht.

Wie schon vor dem Durchbruch mit NGS arbeiten viele Arbeitsgruppen daran, einen praxistauglichen cfDNA-Test für Trisomien auf der Basis von PCR (Polymerasekettenreaktion) $\mathrm{zu}$ etablieren. Dass sich verschiedene PCR,Methoden (z. B. Real-time-PCR, digitale PCR) zum Nachweis fetaler cfDNA eignen, ist schon länger bekannt [14]. Es ist jedoch bis dato nicht gelungen, die Verfahren so weit $\mathrm{zu}$ entwickeln, dass ein robuster, kostengünstiger, kommerziellen Test angeboten werden kann, dessen Performance der auf NGS basierten cfDNATests entspricht. Im Jahr 2015 wurden erste Daten eines auf einer quantitativen, PCR-basierten kommerziellen cfDNATests für Trisomie 21 präsentiert. Unklar ist jedoch, ob die Testgüte dieses Ansatzes, insbesondere seine Spezifität, weiter verbessert und in großen, prospektiven, verblindeten Studien in der allgemeinen Schwangerenpopulation belegt werden kann.

Auch völlig neue, unkonventionelle Ansätze für NIPT wurden bereits vor- gestellt. Schwedischen Wissenschaftlern ist es gelungen, cfDNA in fluoreszierende "DNA-Objekte“ umzuwandeln, die dann mittels Mikrotiterplatte quantifiziert werden können. Dieser Ansatz kommt bemerkenswerterweise völlig ohne aufwendige bioinformatische Datenanalyse und teure Instrumente aus. Der unkomplizierte technische Ansatz soll es auch nicht auf DNA-Analyse spezialisierten Labors ermöglichen NIPT durchzuführen. Unklar bleibt, ob sich bis zur erfolgreichen klinischen Anwendbarkeit solcher Verfahren die Kosten für die Hochdurchsatzsequenzierung nicht bereits soweit verringert haben, dass eine Etablierung im Labor zumindest ökonomisch keinen Sinn mehr ergibt. Klar ist, dass die Kosten für NGS in den letzten 10 Jahren wesentlich schneller gesunken sind, als das Moore-Gesetz es hätte vermuten lassen [15].

\section{Regionalisierung}

Die Durchführung von NIPT war anfangs auf einige wenige Firmen in den USA und China beschränkt, die cfDNATests in ihren eigenen Laboratorien entwickelt und durchgeführt haben. Kurz nach der ersten Kommerzialisierung 2011 haben auch einige Labore außerhalb den USA und China begonnen, cfDNA-Tests lokal zu etablieren. Dabei handelte es sich zumeist um transferierte Technologie der ursprünglichen NIPT-Anbieter. Kommerzielle CE-IVD Kits wurden erstmals im Jahr 2015 angeboten, sie sollen eine großflächige Implementierung von cfDNA-Tests in Laboratorien in ganz Europa und darüber hinaus ermöglichen. Die Regionalisierung von NIPT wird dafür sorgen, dass immer mehr schwangere Frauen Zugang zu cfDNA-Analysen und einer umfangreichen genetischen Abklärung in der Schwangerschaft haben. Die Entwicklung bringt jedoch auch das Risiko einer allgemeinen Verschlechterung der Qualität von NIPT mit sich. Das Anbieten von Tests ohne ausreichende Validierung und die Durchführung von cfDNA-Tests in Laboratorien mit eingeschränkter Erfahrung im Bereich der DNA-Analyse könnten die klinische Performance von NIPT beeinflussen.
Gynäkologe 2016 • 49:437-441

DOI 10.1007/s00129-016-3883-4

(c) The Author(s) 2016

\section{Schmid \\ Nichtinvasive pränatale Tests in der Zukunft. Ausweitung der Indikationen, Evolution der Untersuchungsplattform und Regionalisierung}

\section{Zusammenfassung}

Die Entdeckung, dass zellfreie fetale DNA im mütterlichen Blut existiert und quantifizierbar ist, war die Voraussetzungen für die Entwicklung eines neuen pränatalen Screeningverfahrens. In Zukunft werden sich die Indikationen für nichtinvasive pränatale Tests auf neue genetische Krankheitsbilder ausdehnen und auf kostengünstigen, einfacheren Analyseplattformen regional durchgeführt werden.

\section{Schlüsselwörter}

Chromosomale Anomalien - Desoxyribonukleinsäuren - Schwangerschaft . Humangenetik · Risikoassessment

\section{Noninvasive prenatal testing in the future. Expansion of indications, evolution of the testing platform, and regionalization}

\section{Abstract}

The discovery that cell-free fetal DNA is present in maternal blood and quantifiable led to the development of a new prenatal screening paradigm. In the future, the indications for noninvasive prenatal tests will be expanded to new genetic syndromes and testing will be carried out regionally on cost-effective, simpler analysis-platforms.

\section{Keywords}

Chromosomal aberrations - Deoxyribonucleic acid · Pregnancy · Human genetics . Risk assessment

Die Frage, ob eine fachgerechte cfDNA-Analyse durchgeführt wird, ist meist nicht einfach zu klären. Informationen zu den technischen Hintergründen eines bestimmten cfDNA-Tests und Details zum durchführenden Labor sind für klinische Ärzte oft nur schwer zu erheben. Die Anbieter von NIPT sind daher gefordert, ihre Testmethodik klar $\mathrm{zu}$ beschreiben und ausreichendende 
klinische Validierungsdaten zur Verfügung stellen. Dies geschieht am besten durch Publikation in „peer reviewed journals". Validierungsstudien sind dabei idealerweise prospektive, verblindete Studien mit einem hohen Anteil an Fällen mit vollständigen klinischen Followup. Darüber hinaus sollten diese Daten alle klinisch relevanten Populationen umfassen, einschließlich Studien in der allgemeinen Schwangerenpopulation sowie in Mehrlings- und IVF(In-vitroFertilisation)-Schwangerschaften [16].

Die hohe Genauigkeit von NIPT beruht auf der genauen Beurteilung äußerst geringer Mengen (Nanogramm) von DNA und einer fachgerechten Durchführung der cfDNA-Analyse. Probenkontamination, die zu falsch-negativen und falsch-positiven Ergebnissen führen könnte, muss durch die Implementierung bestimmter baulicher und labortechnischer Maßnahmen so weit wie möglich reduziert werden. Eine Probenverwechslung aufgrund eines menschlichen Fehlers (z. B. Pipettierfehler) kann in der pränatalen Diagnostik schwer wiegende Konsequenzen haben. Dies kann zum Teil durch einen hohen Automatisierungsgrad verhindert werden. Die fachgerechte Durchführung von NIPT erfordert daher meist erhebliche Investitionen, die das Anbieten von cfDNA-Tests in geringen Mengen unwirtschaftlich machen.

\section{》) Der Einsatz eines „point-of- care" NIPT-Tests ist noch nicht absehbar}

Die Etablierung eines „point-of-care“ NIPT-Verfahren, das ohne spezialisiertes Labor auskommt, rasch und kostengünstig verlässliche Ergebnisse direkt in der Ordination liefert, ist derzeit noch nicht absehbar. Die betreuenden Ärzte sind gefordert, das steigende Angebot an NIPT kritisch zu prüfen um die Qualität der Patientenversorgung zu gewährleisten. Darüber hinaus ist es notwendig, dass nationale Behörden angemessene technische und regulatorische Standards für cfDNA-Tests etablieren.

\section{Fazit für die Praxis}

- In Zukunft werden sich die Indikationen für NIPT auf genetische Krankheitsbilder über die klassischen Trisomien hinweg ausdehnen, und die cfDNA-Analyse wird regional auf kostengünstigen, einfacheren Analyseplattformen durchgeführt werden.

- Immer mehr Schwangere werden so Zugang zu cfDNA-Analysemethoden und einer umfangreichen genetischen Abklärung haben.

- Neben vielen Möglichkeiten bringt eine Beurteilung des Risikos für genetische Erkrankungen ohne das Risiko eines invasiven Eingriffs jedoch auch viele ethische, gesellschaftliche und betreuungstechnische Herausforderungen mit sich.

- Nationale Behörden sind gefordert, technische und regulatorische Standards für NIPT zu etablieren.

- Die betreuenden Ärzte sind gefordert, sich über die vielfältigen Möglichkeiten der cfDNA-Analyse zu informieren, um Patienten eine umfassende, wahrhafte und detaillierte Beratung zukommen zu lassen.

- Ethische, ökonomische und medizinische Fragen werden weiterhin die gesellschaftliche Diskussion rund um die klinische Anwendung von NIPT bestimmen.

\section{Korrespondenzadresse}

\section{Assoc. Prof. PD Dr. M. Schmid}

Abteilung für Geburtshilfe und feto-maternale Medizin, Universitätsklinik für Frauenheilkunde, Medizinische Universität Wien, Allgemeines Krankenhaus der Stadt Wien

Währinger Gürtel 18-20, 1090 Wien, Österreich maximilian.schmid@meduniwien.ac.at

\section{Einhaltung ethischer Richtlinien}

Interessenkonflikt. M.Schmid gibt an, dass er Berater von Ariosa Diagnostics, dem Hersteller des Harmony ${ }^{\mathrm{TM}}$ Prenatal Test ist, der Artikel jedoch ausschließlich die persönliche Meinung des Autors wiedergibt.

Dieser Beitrag beinhaltet keine von den Autoren durchgeführten Studien an Menschen oder Tieren.

Open Access. This article is distributed under the terms of the Creative Commons Attribution 4.0 In- ternational License (http://creativecommons.org/ licenses/by/4.0/), which permits unrestricted use, distribution, and reproduction in any medium, provided you give appropriate credit to the original author(s) and the source, provide a link to the Creative Commons license, and indicate if changes were made.

\section{Literatur}

1. Gil MM, Quezada MS, Revello R, Akolekar R, Nicolaides KH (2015) Analysis of cell-free DNA in maternal blood in screening for fetal aneuploidies: updatedmeta-analysis. Ultrasound Obstet Gynecol 45(3):249-266

2. Norton ME, Jacobsson B, Swamy GK, Laurent LC, Ranzini AC, Brar H, Tomlinson MW, Pereira L, Spitz JL, Hollemon D, Cuckle H, Musci TJ, Wapner RJ (2015) Cell-free DNA analysis for noninvasive examination of trisomy. $\mathrm{N}$ Engl J Med 372(17):1589-1597

3. Kitzman JO, Snyder MW, Ventura M, Lewis AP, Qiu $\mathrm{R}$, Simmons LE, Gammill HS, Rubens CE, Santillan DA, Murray JC, Tabor HK, Bamshad MJ, Eichler EE, Shendure J (2012) Noninvasive whole-genome sequencing of a human fetus. Sci Transl Med 4(137): $137 \mathrm{ra76}$

4. Fan HC, Gu W, Wang J, Blumenfeld YJ, ElSayed YY, Quake SR (2012) Non-invasive prenatal measurement of the fetal genome. Nature 487(7407):320-324

5. Schmid M, Klaritsch P, Arzt W, Burkhardt T, Duba HC, Häusler M, Hafner E, Lang U, Pertl B, Speicher M, Steiner H, Tercanli S, Merz E, Heling KS, Eiben B (2015) Cell-Free DNA Testing for Fetal Chromosomal Anomalies in clinical practice: Austrian-German-Swiss Recommendations for non-invasive prenatal tests (NIPT). Ultraschall Med 36(5):507-510

6. Yaron Y, Jani J, Schmid M, Oepkes D (2015) Current status of testing for microdeletion syndromes and rare autosomal trisomies using cell-free DNA technology. Obstet Gynecol 126(5):1095-1099

7. Grati FR, Molina Gomes D, Ferreira JC, Dupont C, Alesi V, Gouas L, Horelli-Kuitunen N, Choy KW, García-Herrero S, Vega AG de la, Piotrowski $K$, Genesio R, Queipo G, Malvestiti B, Hervé B, Benzacken B, Novelli A, Vago P, Piippo K, Leung TY, Maggi F, Quibel T, Tabet AC, Simoni G, Vialard $F$ (2015) Prevalence of recurrent pathogenic microdeletions and microduplications in over 9500 pregnancies. Prenat Diagn 35(8):801-809

8. Wapner RJ, Babiarz JE, Levy B, Stosic M, Zimmermann B, Sigurjonsson S, Wayham N, Ryan A, Banjevic M, Lacroute P, Hu J, Hall MP, Demko Z, Siddiqui A, Rabinowitz M, Gross SJ, Hill M, Benn $P$ (2015) Expanding the scope of noninvasive prenatal testing: detection of fetal microdeletion syndromes. Am J Obstet Gynecol 212(3):332

9. Gross SJ, Stosic M, McDonald-McGinn DM, Bassett AS, Norvez A, Dhamankar R, Kobara K, Kirkizlar E, Zimmermann B, Wayham N, Babiarz JE, Ryan A, Jinnett KN, Demko Z, Benn P (2015) Clinical experience with single-nucleotide polymorphismbased noninvasive prenatal screening for $22 q 11.2$ deletion syndrome. Ultrasound Obstet Gynecol 47(2):177-183

10. Lench $N$, Barrett $A$, Fielding $S$, McKay $F$, Hill M, Jenkins L, White H, Chitty LS (2013) The clinical implementation of non-invasive prenatal diagnosis for single-gene disorders: challenges and progress made. Prenat Diagn 33(6):555-562

11. Skirton H, Goldsmith L, Chitty LS (2015) An easy test but a hard decision: ethical issues concerning non- 
invasive prenatal testing for autosomal recessive disorders. Eur J Hum Genet 23(8):1004-1009

12. Voelkerding KV, Dames SA, Durtschi JD (2009) Next-generation sequencing: from basic research to diagnostics. Clin Chem 55(4):641-658

13. Juneau K, Bogard PE, Huang $S$, Mohseni M, Wang ET, Ryvkin P, Kingsley C, Struble CA, Oliphant A Zahn JM (2014) Microarray-based cell-free DNA analysis improves noninvasive prenatal testing. Fetal Diagn Ther 36(4):282-286

14. Lo YM, Corbetta N, Chamberlain PF, Rai V, Sargent IL, Redman CW, Wainscoat JS (1997) Presence of fetal DNA in maternal plasma and serum. Lancet 350(9076):485-487

15. Wetterstrand K DNA sequencing costs: data from the NHGRI large-scale genome sequencing program. http://www.genome.gov/ sequencingcosts/.Zugegriffen am 19.1.2016

16. Jani J, Rego de Sousa MJ, Benachi A (2015) Cell-free DNA testing: how to choose which laboratory to use? Ultrasound Obstet Gynecol 46(5):515-517

17. Shprintzen RJ (2008) Velo-cardio-facial syndrome: 30 Years of study. Dev Disabil Res Rev 14(1):3-10

18. Shaffer LG, Lupski JR (2000) Molecular mechanisms for constitutional chromosomal rearrangements in humans. Annu Rev Genet 34:297-329

19. Edelmann L, Spiteri E, McCain N, Goldberg R, Pandita RK, Duong S, Fox J, Blumenthal D, Lalani SR, Shaffer LG, Morrow BE (1999) A common breakpoint on 11q23 in carriers of the constitutional $t(11 ; 22)$ translocation. Am J Hum Genet 65(6):1608-1616

20. Battaglia A, Hoyme HE, Dallapiccola B, Zackai E, Hudgins L, McDonald-McGinn D, Bahi-Buisson N, Romano C, Williams CA, Brailey LL, ZuberiSM, Carey JC (2008) Further delineation of deletion 1p36 syndromein 60 patients:arecognizablephenotype and common cause of developmental delay and mental retardation. Pediatrics 121(2):404-410

21. Heilstedt HA, Ballif BC, Howard LA, Lewis RA, Stal $S$, Kashork CD, Bacino CA, Shapira SK, Shaffer LG (2003) Physical map of 1p36, placement of breakpoints in monosomy $1 \mathrm{p} 36$, and clinical characterization of the syndrome. Am J Hum Genet 72(5):1200-1212

22. Gajecka M, Mackay KL, Shaffer LG (2007) Monosomy 1 p36 deletion syndrome. Am J Med Genet C Semin Med Genet 145C(4):346-356

23. Sahoo T, Bacino CA, German JR, Shaw CA, Bird LM, Kimonis V, Anselm I, Waisbren S, Beaudet $A L$, Peters SU (2007) Identification of novel deletions of $15 q 11 q 13$ in Angelman syndrome by array-CGH: molecular characterization and genotype-phenotype correlations. Eur J Hum Genet 15(9):943-949

24. Kim SJ, Miller JL, Kuipers PJ, German JR, Beaudet AL, Sahoo T, Driscoll DJ (2012) Unique and atypical deletions in Prader-Willi syndrome reveal distinct phenotypes. Eur JHum Genet 20(3):283-290

25. Niebuhr E (1978) The Cri du Chat syndrome: epidemiology, cytogenetics, and clinical features. Hum Genet 44(3):227-275

26. Zhang X, Snijders A, Segraves R, Zhang X, Niebuhr A, Albertson D, Yang H, Gray J, Niebuhr E, Bolund L, Pinkel D (2005) High-resolution mapping of genotype-phenotype relationships in cri du chat syndrome using array comparative genomic hybridization. Am J Hum Genet 76(2):312-326

27. Cerruti Mainardi P (2006) Cri du Chat syndrome. Orphanet J Rare Dis 5(1):33

\section{Erratum}

Gynäkologe 2016 · 49:441

DOI 10.1007/s00129-016-3888-z

Online publiziert: 12. Mai 2016

○) Springer-Verlag Berlin Heidelberg 2016. This article is available at SpringerLink with Open Access

(a) CrossMark

\section{J. Pfeiffer ${ }^{1} \cdot$ C. Heinrichs ${ }^{2} \cdot$ J. M. Barinoff' $\cdot$ M. Thill ${ }^{1}$}

' Klinik für Gyäkologie und Geburtshilfe, Zertifiziertes Brustzentrum, AGAPLESION Markus Krankenhaus, Frankfurt am Main, Deutschland

${ }^{2}$ OptiPathR, Gemeinschaftspraxis für Pathologie, Frankfurt am Main, Deutschland

\section{Erratum zu: Suspekte Endometriumhyperplasie}

\author{
Erratum zu: \\ Der Gynäkologe 2016 \\ DOI: $10.1007 / \mathrm{s} 00129-016-3842-0$
}

Wir möchten Sie darauf hinweisen, dass in diesem Artikel der Letztautor PD Dr. M. Thill aufgrund eines Fehlers im Produktionsprozess nicht aufgeführt wurde. Bitte beachten Sie die richtige Autorenschaft: J. Pfeiffer, C. Heinrichs, J.M. Barinoff, $\mathrm{M}$. Thill

Wir bitten, den Fehler zu entschuldigen.

Die Redaktion

\section{Korrespondenzadresse}

PD Dr. M. Thill

Klinik für Gyäkologie und Geburtshilfe, Zertifiziertes Brustzentrum, AGAPLESION Markus Krankenhaus Wilhelm-Epstein-Straße 4, 60431 Frankfurt am Main, Deutschland marc.thill@fdk.info
Die Online-Version des Originalartikels ist unter doi:10.1007/s00129-016-3842-0 zu finden. 